\title{
Role of thymus on prognosis of myasthenia gravis in Turkish population
}

\author{
(1) Hulya Tireli, ${ }^{1}$ (i) Gulbun Yuksel, ${ }^{1}$ () Tamer Okay, ${ }^{2}$ (ㅇ) Kemal Tutkavul ${ }^{1}$ \\ ${ }^{1}$ Department of Neurology, Haydarpasa Numune Training and Research Hospital, Istanbul, Turkey \\ ${ }^{2}$ Department of Thorax Surgery, Dr. Siyami Ersek Thoracic and Cardiovascular Surgery Training and Research Hospital, Istanbul, Turkey
}

\begin{abstract}
OBJECTIVE: Myasthenia gravis (MG) is an autoimmune disease that may cause a disorder in transmission at the neuromuscular junction. Antibodies directed against acetylcholine receptors are responsible. The thymus is the place that that production of these antibodies mainly occurs. The thymus gland abnormalities and abnormal production of these antibodies are associated with MG. Consequently, thymectomy is a common treatment for MG. The nature of the disease makes it difficult to plan prospective, controlled trials; therefore, there is no current consensus among clinicians on a single algorithm of treatment, and the approach is frequently based on the observations and experiences of experts. The contributions to the literature largely consist of retrospective studies examining an approach to treatment and the effects of thymectomy on prognosis. In this retrospective study, evaluation of Turkish patients with myasthenia gravis was carried out for the importance of thymectomy and effects on prognosis.
\end{abstract}

METHODS: In this study, 93 patients with myasthenia gravis whose followed up at Neuromuscular outpatient clinic between 1998-2018 were evaluated retrospectively. Type of disease, antibody status, treatment, thymectomy, thymus pathology and prognosis were assessed.

RESULTS: Thymectomy had been a positive effect on the prognosis of the disease independent of the duration of disease and thymic pathology. The best results had been obtained with early thymectomy with short disease duration, younger age and patients with thymic hyperplasia. Success of therapy was limited with thymoma. With advanced age need for thymectomy was decreased.

CONCLUSION: In the present study, evaluation of 93 patients with myasthenia gravis was done retrospectively and it was concluded that thymectomy had a positive effect on prognosis, especially in young patients when performed as early as possible. The most successful results were obtained in cases with thymic hyperplasia.

Keywords: Myasthenia gravis; prognosis; thymectomy.

Cite this article as: Tireli H, Yuksel G, Okay T, Tutkavul K. Role of thymus on prognosis of myasthenia gravis in Turkish population. North Clin Istanb 2020;7(5):452-459.

$\mathrm{M}$ yasthenia gravis $(\mathrm{MG})$ is an autoimmune disease caused by a disruption of neuromuscular transmission, a result of antibodies directed against acetylcholine receptors (AChR) on the postsynaptic membrane at the neuromuscular junction. Local or generalized muscle weakness, which may fluctuate during the day, is a typical feature. In cases where the bulbar and respiratory muscles are involved, a myasthenic crisis may occur, and potentially fatal respiratory failure is seen in some $15 \%$ of all patients with myasthenia gravis. Although it is known as a disease of the young, it also appears in the sixth and seventh decades [1-3].

Received: September 29, 2019 Accepted: April 29, 2020 Online: August 10, 2020

Correspondence: Hulya TIRELI, MD. Saglik Bilimleri Universitesi, Haydarpasa Numune Egitim ve Arastirma Hastanesi, Noroloji Klinigi, Istanbul, Turkey.

Tel: +902165423232 e-mail: hulyatireli@hotmail.com

(c) Copyright 2020 by Istanbul Provincial Directorate of Health - Available online at www.northclinist.com 
Autoantibodies developed against AChR may lead to complement-related damage in the postsynaptic membrane, and AChR loss results in weakness and easy fatiguability. Anti-tyrosine kinase muscle-specific kinase (MuSK) and anti-low-density lipoprotein receptor-related protein (LRP4) antibodies have been detected in patients without AChR antibodies [4-6].

Although the muscle weakness is generally symmetrical, ocular muscle involvement may also be asymmetrical. Muscle weakness occurring with repeated movements and fatigue caused by exercise may vary during the day and from day to day $[1,3]$.

The diagnosis of MG is based on typical symptoms and findings, as well as the detection of specific antibodies. Most patients have AChR antibodies. In others, anti-MuSK antibodies and LRP4 antibodies are detected. An ice-pack test, in addition to pharmacological and neurophysiological tests, is helpful in the diagnosis of patients without antibodies. The condition of the thymus can be evaluated with mediastinal imaging [7].

The thymus has been known to play a role in the pathogenesis of MG for many years. After adolescence, ordinarily, the thymus gland gradually atrophies in adulthood and is almost lost in the mediastinal tissue. In patients with $\mathrm{MG}$, the expected involution of the thymus tissue does not take place, and pathological changes, such as thymic hyperplasia or thymoma, may occur.

After diagnosis, treatment often consists of symptomatic relief with acetylcholinesterase inhibitors, immunosuppressive agents, and thymectomy. The primary goal is to achieve complete or near-complete pharmacological remission. That is, attaining significant reduction or elimination of myasthenic symptoms and effects can be defined as successful treatment. Mild eye involvement may be considered acceptable [1].

Many immunosuppressive and immunomodulatory drugs are used in the treatment of MG, and new drugs are regularly added to the list. However, as yet, there is no clinically accepted standard treatment. Since large, randomized trials are difficult to perform due to the nature of the disease, there is no current consensus on treatment [8]. Therefore, clinical treatment approaches are largely based on the evaluation of large case series and expert approaches [3].

This study was an evaluation of the effects of thymectomy on the prognosis of MG patients.

\section{MATERIALS AND METHODS}

This study was a retrospective evaluation of the data of 93 patients with MG who were followed up at the Neuromuscular Diseases outpatient clinic between 1998 and 2018. Health Sciences University Haydarpasa Training and Research Hospital Scientific Committee approved this study on 28.08.2018 with reference number of $771 / 08 / 2018$.

The study population consisted of $56(60.2 \%)$ female and $37(39.8 \%)$ male patients. The mean age of onset was $46.49 \pm 16.38$ years. In all, $81.7 \%$ of the patients were grouped as generalized MG (gMG), while the remainder was categorized as ocular $\mathrm{MG}(\mathrm{OMG})$. The patients were also grouped according to the age of onset of the disease: early-onset was defined as before 50 years of age $(n=56,60.2 \%)(E O M G)$, and patients in whom MG was diagnosed after the age of $50(n=37,39.8 \%)$ were described as late-onset (LOMG). Of the total of $76 \mathrm{gMG}$ patients, 54 underwent thymectomy and 22 patients did not. Two of oMG patients underwent thymectomy because the thymoma was reported in thorax CT. A test for the AChR antibody was positive in 53 patients (67.9\%) and negative in 25 patients (32.1\%).

Of the patients with gMG, in $74 \%$, a thymectomy was performed as soon as possible after diagnosis. Symptomatic control was achieved with an acetylcholinesterase inhibitor first, and then intravenous immunoglobulin (IVIg) was administered in preparation for the operation. The operation was performed once the patient was in a clinically stable condition. Immunosuppressive treatment was implemented quickly in the postoperative follow-up period. Corticosteroids $(1 \mathrm{mg} / \mathrm{kg})$ were administered for immunosuppressive purposes, combined with azathioprin (AZA; $2 \mathrm{mg} / \mathrm{kg}$ ), to prevent possible side effects of corticosteroids. When corticosteroid side effects occurred while using this immunosuppressive combination, the use of the corticosteroid was discontinued and immunosuppression was achieved with AZA alone to prevent any long-term adverse effects of corticosteroids. Minimal complications or adverse effects were seen with this treatment regimen. In addition, regular IVIg, plasma exchange therapy, cyclophosphamide, mycophenolate mofetil or rituximab treatment was also used in patients whose symptoms were under control.

The thymectomies were performed by a single surgical team at one center as a complete and extended thymectomy with a partial or complete sternotomy. 
In all, 54 gMG and 2 oMG patients underwent thymectomy. Thymoma was detected in 20 (36.4\%), thymic hyperplasia in $21(38.2 \%)$, and thymic involution in $14(25.5 \%)$ patients.

The scoring system recommended by the Myasthenia Gravis Foundation of America (MGFA) was used to determine the clinical level of disease during the first and final evaluation of treatment. A number between 1 and 4 is used to score the patient's condition. The absence of any complaint was evaluated as full recovery and an improvement in the MGFA score following treatment was considered a partial recovery.

\section{Statistical Analysis}

NCSS 2007 (NCSS, Inc., Kaysville, UT, USA) software was used to perform the statistical analysis. Descriptive statistical methods (mean, SD, median, frequency, ratio, minimum, maximum) were applied. Pearson's chi-square test, the Fisher-Freeman-Halton test, and Fisher's exact test were used to compare qualitative data. The Wilcoxon signed-rank test was used for intragroup comparisons of non-normally distributed parameters. Significance was established at $\mathrm{p}<0.05$.

\section{RESULTS}

In this study, the treatment approach used in 93 patients with MG and the results observed after thymectomy were evaluated retrospectively.

Among these 93 patients, the gMG group consisted of 76 patients ( 49 females, 27 males) with a mean age of $46.49 \pm 16.38$ years (min-max: $12-88$ years). In this group, 45 patients tested positive for $\mathrm{AChR}$ antibodies, while 19 patients had a negative result for the AChR antibody. The results of 12 patients could not be obtained. Twelve patients were examined for anti-MuSK antibodies and a positive result was seen in three cases. Thymectomy was performed as soon as possible in patients with early-onset disease that was of short duration. oMG and gMG patients of all ages whose thorax CT revealed thymoma also underwent thymectomy.

The gMG patients who did and did not undergo thymectomy were compared. Of the 76 patients in the gMG group, 53 patients were treated with a thymectomy and 22 patients did not undergo thymectomy. Only six of the 22 patients who did not undergo thymectomy were under 50 years of age at the onset of the disease. Among the patients who did not undergo thymecto- my, seven patients $(30.4 \%)$ had complete recovery, four (17.4\%) patients experienced partial improvement, 11 patients $(47.8 \%)$ did not demonstrate any change, and the clinical condition of one patient (4.3\%) deteriorated. Patients who did not show any change compared with their initial clinical presentation or whose clinical condition worsened constituted more than half $(52.1 \%)$ of the non-thymectomized gMG group, while $47.8 \%$ of the patients experienced complete or partial improvement $(\mathrm{p}=0.010 ; \mathrm{p}<0.05)$.

Among the thymectomized patients, 21 (39.6\%) patients had a complete recovery, 12 patients $(22.6 \%)$ showed an improvement, the clinical condition of 18 patients $(34 \%)$ did not change, and it worsened in two cases $(3.8 \%)$. In this group, $62.2 \%$ of the patients recovered completely or partially, while the clinical condition of $37.8 \%$ remained the same or worsened $(p=0.001$; $\mathrm{p}<0.01$ ). Greater improvement was observed in the mildly generalized thymectomy group when compared with patients who did not undergo the procedure. Thymectomy had a positive effect on prognosis, independent of the type of pathology or duration of the disease (Table 1 ).

Twenty-seven (50\%) of the gMG patients underwent thymectomy within the first six months of diagnosis, while $15(27.7 \%)$ and $12(22.2 \%)$ patients were operated within seven to 12 months, and more than a year after the establishment of the diagnosis, respectively. Among those who underwent thymectomy within six months, a complete recovery was observed in $44.4 \%, 14.8 \%$ of them showed an improvement, there was no change in $33 \%$, and the condition of $7.4 \%$ deteriorated $(p=0.001$; $\mathrm{p}<0.01)$. Fifteen $(27.7 \%)$ patients underwent thymectomy within 7 to 12 months after diagnosis. Among these patients, $20 \%$ demonstrated complete recovery, and $40 \%$ had some improvement, while the clinical condition of $40 \%$ did not change $(p=0.008 ; p<0.01)$. As a result, thymectomy latency was associated with complete recovery in $44.4 \%$ of the patients who underwent the procedure within six months and $20 \%$ of the patients who underwent the operation within 7-12 months after diagnosis. An earlier thymectomy was beneficial to prognosis (Table 2).

Thymectomy was performed in 12 patients $(22.2 \%)$ more than one year after the diagnosis. Complete recovery was achieved in $58.3 \%$, and $16.7 \%$ of these patients experienced a partial recovery, while the clinical condition of $25 \%$ did not change significantly $(p=0.005 ; p<0.01)$. In this group, the level of disease observed was less se- 
TABLE 1. Assessment of the MGFA scores according to thymectomy on generalised MG

\begin{tabular}{|c|c|c|c|c|c|}
\hline & \multicolumn{2}{|c|}{$\begin{array}{l}\text { Thymectomy }(+) \\
(n=53)\end{array}$} & \multicolumn{2}{|c|}{$\begin{array}{l}\text { Thymectomy }(-) \\
(n=23)\end{array}$} & \multirow[b]{2}{*}{$\mathrm{p}$} \\
\hline & $\mathrm{n}$ & $\%$ & $n$ & $\%$ & \\
\hline \multicolumn{6}{|c|}{ MGFA scores at onset } \\
\hline 2 & 39 & 73.6 & 16 & 69.6 & a 0.719 \\
\hline $3+4$ & 14 & 26.4 & 7 & 30.4 & \\
\hline \multicolumn{6}{|c|}{ MGFA scores at the end } \\
\hline $0+1$ & 26 & 49.1 & 9 & 39.1 & b0.624 \\
\hline 2 & 23 & 43.4 & 13 & 56.5 & \\
\hline \multirow[t]{2}{*}{$3+4$} & 4 & 7.5 & 1 & 4.3 & \\
\hline & \multicolumn{2}{|c|}{$0.001 * *$} & \multicolumn{2}{|c|}{$0.010 *$} & \\
\hline \multicolumn{6}{|c|}{ Recovery according to MGFA scores at onset and at the end } \\
\hline Comp recovery & 21 & 39.6 & 7 & 30.4 & b0.568 \\
\hline Part recovery & 12 & 22.6 & 4 & 17.4 & \\
\hline Non difference & 18 & 34.0 & 11 & 47.8 & \\
\hline Deterioration & 2 & 3.8 & 1 & 4.3 & \\
\hline
\end{tabular}

MGFA: Myasthenia Gravis Foundation of America; a: Pearson Ki-kare Test; b: Fisher-Freeman-Halton Test; c: Wilcoxon Signed-Ranks Test; *: $\mathrm{p}<0.05$; **: $\mathrm{p}<0.01$. Thymectomy had a positive effect on prognosis, independent of the type of pathology or duration of the disease.

TABLE2. Assessment of the MGFA scores according to operation latency on generalised MG

\begin{tabular}{|c|c|c|c|c|c|c|c|}
\hline & \multicolumn{6}{|c|}{ Operation latency } & \multirow[b]{3}{*}{$\mathrm{p}$} \\
\hline & \multicolumn{2}{|c|}{$\begin{array}{l}1-6 \text { mo } \\
(n=27)\end{array}$} & \multicolumn{2}{|c|}{$\begin{array}{c}7-12 \mathrm{mo} \\
(\mathrm{n}=15)\end{array}$} & \multicolumn{2}{|c|}{$\begin{array}{l}>13 \text { mo } \\
(n=12)\end{array}$} & \\
\hline & $\mathrm{n}$ & $\%$ & $\mathrm{n}$ & $\%$ & $\mathrm{n}$ & $\%$ & \\
\hline \multicolumn{8}{|c|}{ MGFA scores at onset } \\
\hline 2 & 22 & 81.5 & 8 & 53.3 & 10 & 83.3 & b0.125 \\
\hline $3+4$ & 5 & 18.5 & 7 & 46.7 & 2 & 16.7 & \\
\hline \multicolumn{8}{|c|}{ MGFA scores at the end } \\
\hline $0+1$ & 14 & 51.9 & 4 & 26.7 & 9 & 75.0 & bo.105 \\
\hline 2 & 10 & 37.0 & 10 & 66.7 & 3 & 25.0 & \\
\hline \multirow[t]{2}{*}{$3+4$} & 3 & 11.1 & 1 & 6.7 & 0 & 0 & \\
\hline & \multicolumn{2}{|c|}{$0.001 * *$} & \multicolumn{2}{|c|}{$0.008 * *$} & \multicolumn{2}{|c|}{$0.005 * *$} & \\
\hline \multicolumn{8}{|c|}{ Recovery according to MGFA scores at onset and at the end } \\
\hline Comp recovery & 12 & 44.4 & 3 & 20.0 & 7 & 58.3 & b0.277 \\
\hline Part recovery & 4 & 14.8 & 6 & 40.0 & 2 & 16.7 & \\
\hline Non difference & 9 & 33.3 & 6 & 40.0 & 3 & 25.0 & \\
\hline Deterioration & 2 & 7.4 & 0 & 0 & 0 & 0 & \\
\hline
\end{tabular}

MGFA: Myasthenia Gravis Foundation of America; b: Fisher-Freeman-Halton Test; c: Wilcoxon Signed-Ranks Test; **: p<0.01. The difference between MGFA scores at onset and at the end was found statistically significant in patient group whose operation latency was between 1-6 mo. Similar results were obtained in 7-12 mo and $>13$ mo groups. $44.4 \%$ of patients had complete recovery versus $20 \%$ of patients, $1-6$ mo operation latency versus $7-12$ mo operation latency, respectively. 
TABLE 3. Assessment of the MGFA scores according to thymic pathology on generalised MG

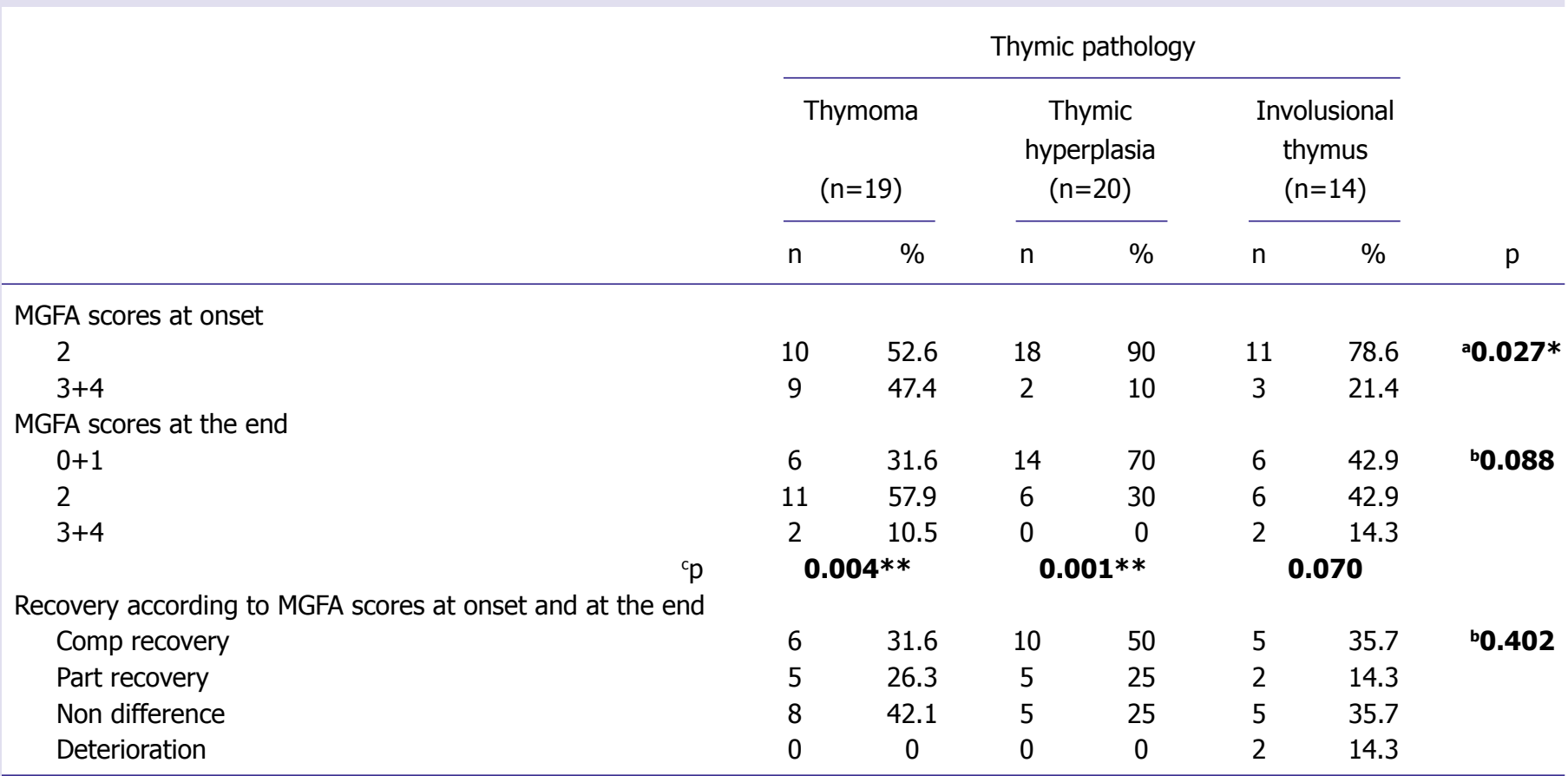

MGFA: Myasthenia Gravis Foundation of America; a: Pearson Ki-kare Test; b: Fisher-Freeman-Halton Test; c: Wilcoxon Signed-Ranks Test; **: $\mathrm{p}<0.01 ; *$ : $\mathrm{p}<0.05$. In patients with thymoma and thymic hyperplasia, there was significant difference between MGFA scores at onset and at the end of assessment, ( $p=0.004, p=0.001$, respectively). In patients with thymic hyperplasia complete recovery was more significant ( $31.6 \%$ versus $50 \%$ ).

vere. Resolution of complaints was observed in $58.3 \%$. The delayed thymectomy in this group was the result of a belated diagnosis due to a benign course and later presentation. A different antigenic status may also have played a role in the pathogenesis of this group.

Of the 53 gMG patients who underwent thymectomy, the results revealed 19 (35\%) cases of thymoma, 20 (37\%) patients with thymic hyperplasia, and $14(25.9 \%)$ patients with a normal thymus and involutional changes. Of the 19 patients with thymoma, 16 patients were under 50 years of age. The incidence of thymoma decreases with advanced age. The pretreatment clinical manifestations of the patients with thymoma were more severe than patients with hyperplasia $(p=0.027 ; p<0.05)$ Complete improvement was observed in $31.6 \%$ and partial improvement was seen in $26.6 \%$ of the patients with thymoma after treatment, while in $42.1 \%$ of the patients, we did not see improvement $(\mathrm{p}=0.004 ; \mathrm{p}<0.01)$.

Complete recovery was seen in $50 \%$ of the patients with thymic hyperplasia, and some improvement was recorded in $25 \%(\mathrm{p}=0.001 ; \mathrm{p}<0.01)$. Complete or partial improvement was observed in $35.7 \%$ and $14.3 \%$, re- spectively, of the cases with thymus involution $(\mathrm{p}=0.070$; $\mathrm{p}>0.05$ ) After thymectomy, the clinical condition of $\mathrm{pa}$ tients in all three groups improved. The best prognosis was seen in $50 \%$ of the patients with thymic hyperplasia and $31 \%$ of the patients with thymoma. The presence of thymoma limits potential improvement (Table 3).

There was a statistically significant difference in the length of time until surgery according to the presence of thymoma in patients undergoing thymectomy $(p=0.009$; $\mathrm{p}<0.01$ ). The time to thymectomy (latency) was more frequently between one and six months in cases with thymoma, while it was often 13 months or more in non-thymomatous patients.

Myasthenic crisis was observed in $29 \%(n=28)$ of the study group. The pathology was thymoma in 14 cases in this group (50\%), involution and normal thymus in seven (25\%), and thymic hyperplasia in two (7.14\%). Thymoma was detected in $21(21.8 \%)$ of the total group of 96 patients. A myasthenic crisis was observed in $14(66.6 \%)$ of these patients. The incidence of myasthenic crisis was significantly higher in thymomatous patients. 


\section{DISCUSSION}

Myasthenia Gravis is the most frequently seen neuromuscular junction disease. AChR antibodies are often detected in patients with MG, and anti-MuSK, anti-titin, LRP4 antibodies, and ryanodine antibodies may also be found. Generally, thymectomy in the early stages of the disease is recommended for all $\mathrm{gMG}$ patients because the thymus is thought to be responsible for the pathogenesis of the disease [9]. gMG patients often produce antibodies against $\mathrm{AChR}$ and these patients respond well to thymectomy. In recent years, investigators have not recommended a thymectomy for patients with LRP4 antibodies because the disease has different pathogenesis and is seen in patients without AChR and MuSK antibodies, so it may, in fact, be considered a different disease. Patients with LRP4 antibodies generally have a mild disease course that may manifest with a clinical picture resembling pure oMG. Recently, LRP4 antibodies have been detected in MG patients with both AChR antibodies and MuSK antibodies, as well as other autoimmune diseases and amyotrophic lateral sclerosis [3].

AChR antibody-positivity was detected in 41 of our patients while anti-MuSK antibody-positivity in three patients. The presence of LRP4 antibodies was not investigated.

In our patient group, complete or partial recovery was observed in $47.8 \%$ of the patients who did not undergo thymectomy but received immunosuppressive treatment, while the complete or partial recovery was observed in $62.2 \%$ of patients who underwent thymectomy. As observed in our study, the positive effects of thymectomy on clinical outcome have been reported in various studies [2].

An early thymectomy can be protective against AChR-specific T-cells exiting the thymus and entering the lymph nodes and peripheral lymphoid tissue. Removing the entire thymus is preventative $[2,10,11]$.

In our study group, thymectomy was performed in 53 of $74 \mathrm{gMG}$ patients; in all, $49 \%$ were operated on within the first six months after the onset of the disease. In this group, $44.4 \%$ of the patients had a complete recovery. The percentage of complete recovery decreased to $20 \%$ of the patients who underwent thymectomy within seven to 12 months. This result supports the idea that an early thymectomy may play a role in the disruption of the immune cascade.

Thymectomy is recommended in all MG cases with thymoma. In addition, thymectomy is also recommend- ed for immune modulation in patients without thymoma. Especially in cases with early-onset MG, thymectomy improves the clinical course and decreases the need for immunosuppressive drugs [12].

In most thymoma patients, thymectomy latency is between one and six months. This may be due to the onset of severe symptoms in patients with thymoma, which may lead to earlier medical visits. In our patient group, the pretreatment clinical symptoms of the thymoma patients were more severe when compared with those of the thymic hyperplasia patients. Myasthenic crisis is more frequently seen in patients with thymoma.

Several methods are used to perform thymectomy. In addition to partial or complete sternotomy techniques, there are also less invasive methods, such as video- or robot-assisted mediastinoscopic thymectomy [2]. An extended transsternal thymectomy provides maximal resection of thymic tissue with a low risk of phrenic nerve damage or other morbidity. Some $85 \%$ to $95 \%$ of thymic tissue can be resected using this method [13]. Less invasive thymectomy approaches are often preferred due to the similar efficacy, shorter postoperative recovery time, and better cosmetic results $[14,15]$. However, there is a possibility of leaving ectopic thymic tissue behind in perithymic or pericardial areas with a minimally invasive technique [16]. In some studies, ectopic thymus tissue has been reported to have negative effects on the outcome [17]. It has also been reported that an incomplete thymic resection resulted in no clinical improvement $[18$, 19]. When the advantages and disadvantages of a less invasive thymectomy and more aggressive treatment were compared, the extended transsternal technique demonstrated a better prognosis. In fact, the largest possible resection has been recommended. In a randomized, multicenter study of 126 patients, Wolfe [20] reported that an extended thymectomy improved the clinical outcome and reduced the need for immunosuppressive therapy.

A transsternal extended thymectomy was performed in all cases in this study; there was homogenization concerning operation technique.

Several studies have investigated the prognosis of patients with MG with thymoma. In some studies, it has been indicated that the presence of thymoma adversely affected prognosis, but other authors have stated that patients with thymic carcinoma in association with $\mathrm{MG}$ had a better prognosis $[21,22]$. Some researchers have also concluded that thymoma did not cause any significant change in an MG prognosis [23]. 
When our thymectomized patients were grouped for a response to thymectomy according to thymus pathology, $31 \%$ of the patients with thymoma, $50 \%$ of the patients with thymic hyperplasia, and $35.7 \%$ of the patients with normal thymic tissue were seen a complete recovery. The best improvement was seen in thymic hyperplasia patients, which is similar to some literature results. There is speculation that hyperplastic thymus contributes to the production of AChR antibodies and that in MG patients with thymoma, it may be produced in the peripheral lymphoid tissue [24] Siwachat et al. [25] reported greater improvement with thymectomy in patients with thymic hyperplasia, similar to our results.

In summary, in patients with gMG, a thymectomy is preferable if the patient is young and the disease duration is short. In cases with hyperplastic thymus, greater improvement is seen when a thymectomy is performed at an early stage of the disease. The best results are observed in patients with thymic hyperplasia. The presence of thymoma reduces the chances of improvement. Thymectomy has a positive effect on prognosis regardless of the thymic pathology or duration of the disease. Extended thymectomy is a preferred method because it is less likely to leave residual thymus tissue. The course of MG also changes with age. Thymoma is less common and the disease leads a milder course in older patients, which suggests that the elderly group may have a different antigenic structure.

Acknowledgements: We thank to Gizem Gursoy MD for documentation, Bulent Aydemir MD and Oya Imamoglu Uncu MD for performing the surgeries. We also thank to Ayse Akpinar MD for follow up some of the patients.

Ethics Committee Approval: The Haydarpasa Numune Training and Research Hospital Scientific Committee granted approval for this study (date: 28.08.2018, number: 771/08/2018).

Conflict of Interest: No conflict of interest was declared by the authors.

Financial Disclosure: The authors declared that this study has received no financial support.

Authorship Contributions: Concept - HT, ; Design - HT, GY; Supervision - HT, KT; Fundings - HT; Materials - HT; Data collection and/or processing - GY, TO; Analysis and/or interpretation - HT, GY; Literature review - HT, KT; Writing - HT, KT; Critical review - KT.

\section{REFERENCES}

1. Gilhus NE. Myasthenia Gravis. N Engl J Med 2016;375:2570-81.

2. Gilhus NE, Verschuuren JJ. Myasthenia gravis: subgroup classification and therapeutic strategies. Lancet Neurol 2015;14:1023-36. [CrossRef]

3. Gilhus NE, Skeie GO, Romi F, Lazaridis K, Zisimopoulou P, Tzartos
S. Myasthenia gravis - autoantibody characteristics and their implications for therapy. Nat Rev Neurol 2016;12:259-68. [CrossRef]

4. Phillips WD, Vincent A. Pathogenesis of myasthenia gravis: update on disease types, models, and mechanisms. F1000Res 2016;5:F1000 Faculty Rev-1513. [CrossRef]

5. Shen C, Lu Y, Zhang B, Figueiredo D, Bean J, Jung J, et al. Antibodies against low-density lipoprotein receptor-related protein 4 induce myasthenia gravis. J Clin Invest 2013;123:5190-202. [CrossRef]

6. Huijbers MG, Zhang W, Klooster R, Niks EH, Friese MB, Straasheijm $\mathrm{KR}$, et al. MuSK IgG4 autoantibodies cause myasthenia gravis by inhibiting binding between MuSK and Lrp4. Proc Natl Acad Sci U S A 2013;110:20783-8. [CrossRef]

7. Jordan B, Kellner J, Jordan K, Bähre M, Behrmann C, Zierz S. Thymic pathologies in myasthenia gravis: a preoperative assessment of CAT scan and nuclear based imaging. J Neurol 2016;263:641-8. [CrossRef]

8. Sanders DB, Wolfe GI, Narayanaswami P; MGFA Task Force on MG Treatment Guidance. Developing treatment guidelines for myasthenia gravis. Ann N Y Acad Sci 2018;1412:95-101. [CrossRef]

9. Conti-Fine BM, Diethelm-Okita B, Ostlie N, Wang W, Milani M. Immunopathogenesis of myasthenia gravis. In: Kaminski HJ, editor. Myasthenia Gravis and related disorders. NewYork: Humana Press; 2009. p. 43-70. [CrossRef]

10. Gronseth GS, Barohn RJ. Practice parameter: thymectomy for autoimmune myasthenia gravis (an evidence-based review): report of the Quality Standards Subcommittee of the American Academy of Neurology. Neurology 2000;55:7-15. [CrossRef]

11. Marx A, Pfister F, Schalke B, Saruhan-Direskeneli G, Melms A, Ströbel $P$. The different roles of the thymus in the pathogenesis of the various myasthenia gravis subtypes. Autoimmun Rev 2013;12:875-84. [CrossRef]

12. Rückert JC, Swierzy M, Kohler S, Meisel A, Ismail M. Thymectomy in Myasthenia Gravis. Neurology International Open 2018;2:E124-30.

13. Jaretzki A $3^{\text {rd }}$, Barohn RJ, Ernstoff RM, Kaminski HJ, Keesey JC, Penn AS, et al. Myasthenia gravis: recommendations for clinical research standards. Task Force of the Medical Scientific Advisory Board of the Myasthenia Gravis Foundation of America. Neurology 2000;55:16-23.

14. Lee CY, Kim DJ, Lee JG, Park IK, Bae MK, Chung KY. Bilateral videoassisted thoracoscopic thymectomy has a surgical extent similar to that of transsternal extended thymectomy with more favorable early surgical outcomes for myasthenia gravis patients. Surg Endosc 2011;25:84954. [CrossRef]

15. Rückert JC, Swierzy M, Ismail M. Comparison of robotic and nonrobotic thoracoscopic thymectomy: a cohort study. J Thorac Cardiovasc Surg 2011;141:673-7. [CrossRef]

16. Jaretzki A $3^{\text {rd }}$. Thymectomy for myasthenia gravis: analysis of the controversies regarding technique and results. Neurology 1997;48:S5263. [CrossRef]

17. Ponseti JM, Gamez J, Vilallonga R, Ruiz C, Azem J, López-Cano M, et al. Influence of ectopic thymic tissue on clinical outcome following extended thymectomy in generalized seropositive nonthymomatous myasthenia gravis. Eur J Cardiothorac Surg 2008;34:1062-7. [CrossRef]

18. Jaretzki A $3^{\text {rd }}$, Penn AS, Younger DS, Wolff M, Olarte MR, Lovelace RE, et al."Maximal" thymectomy for myasthenia gravis. Results. J Thorac Cardiovasc Surg 1988;95:747-57. [CrossRef]

19. Miller RG, Filler-Katz A, Kiprov D, Roan R. Repeat thymectomy in chronic refractory myasthenia gravis. Neurology 1991;41:923-4.

20. Wolfe GI, Kaminski HJ, Aban IB, Minisman G, Kuo HC, Marx A, et al; MGTX Study Group. Randomized Trial of Thymectomy in Myasthenia Gravis. N Engl J Med 2016;375:511-22. [CrossRef]

21. Li W, Miao Z, Liu X, Zhang Q, Sun L, Li P, et al. Thymic carcinoma 
patients with myasthenia gravis exhibit better prognoses. Int J Clin Oncol 2016;21:75-80. [CrossRef]

22. Zhang Z, Cui Y, Jia R, Xue L, Liang H. Myasthenia gravis in patients with thymoma affects survival rate following extended thymectomy. Oncol Lett 2016;11:4177-82. [CrossRef]

23. Cacho-Díaz B, Salmerón-Moreno K, Lorenzana-Mendoza NA, Texcocano J, Arrieta O. Myasthenia gravis as a prognostic marker in patients with thymoma. J Thorac Dis 2018;10:2842-8. [CrossRef]
24. Zheng Y, Cai YZ, Shi ZY, Qiu Y, Zhang HL, Wang ZH, et al. Different neurologic outcomes of myasthenia gravis with thymic hyperplasia and thymoma after extended thymectomy: A single center experience. J Neurol Sci 2017;383:93-8. [CrossRef]

25. Siwachat S, Tantraworasin A, Lapisatepun W, Ruengorn C, Taioli E, Saeteng S. Comparative clinical outcomes after thymectomy for myasthenia gravis: Thoracoscopic versus trans-sternal approach. Asian J Surg 2018;41:77-85. [CrossRef] 